\title{
Dos tipos de construcciones con si en italiano
}

\author{
Patrizia Romani
}

Universidad Autónoma del Estado de México

paromani@libero.it

\begin{abstract}
El trabajo analiza, recurriendo a la teoría generativista de Rección y Ligamiento, dos grandes clases de construcciones del italiano con el clítico si («se»): las que tienen un valor impersonal e implican la intervención de un agente indeterminado necesariamente humano, y aquéllas que, como las reflexivas y las medias, implican la existencia de un agente correferencial con el único participante expresado. Este segundo grupo se caracteriza por la presencia de un si anafórico, mientras que en el primero interviene un si no anafórico. Cualquiera que sea su significado, las construcciones con el si carecen todas de un sujeto profundo temático. Los dos si, el anafórico y el no anafórico, constituyen una única entidad que sólo posee el rasgo [+Persona], la tercera, y ningún otro contenido referencial; se generan en el mismo lugar estructural y condicionan del mismo modo el comportamiento sintáctico del núcleo Flex; su única diferencia radica en la relación de coindización que el clítico puede o no establecer con la categoría que funciona como sujeto superficial.
\end{abstract}

Palabras clave: si reflexivo, si medio, si impersonal, pronombre clítico, correferencia.

\section{Abstract}

This article uses Government and Binding Theory to analyze two groups of Italian constructions with the clitic si: those which have an impersonal meaning and imply the existence of an indeterminate agent that is necessarily human, and those which imply the existence of an agent that is coreferent with the one expressed. This second group of constructions presents an anaphoric si; on the contrary, a non-anaphoric si appears in the first one. Whatever meaning they may have, none of the constructions with a clitic si has a thematic deep subject. Both the anaphoric and the non-anaphoric si, constitute a single entity that has the $\left[+3^{\text {rd }}\right.$ Person] feature, with no further referential content. The two si are generated in the same structural place and they influence the syntactic behaviour of the node INFL in the same way. Their only difference lies in the relationship of coreference that the clitic si may or may not establish with the superficial subject.

Key words: reflexive $s i$, ergative $s i$, impersonal si, clitic pronoun, coreference. 


\section{Introducción}

Objetivo del presente trabajo es analizar, recurriendo a la teoría generativista de Rección y Ligamiento, dos grandes clases de construcciones del italiano con el clítico si («se»): las que tienen un valor impersonal e implican la existencia de un agente indeterminado necesariamente humano, y aquéllas que, como las reflexivas y las medias, implican la intervención de un agente correferencial con el único participante expresado. Este segundo grupo se caracteriza por la presencia de un $s i$ anafórico, mientras que en el primero interviene un si no anafórico.

Cualquiera que sea su significado, las construcciones con el si carecen todas de un sujeto profundo temático, según se verá a continuación.

\section{Las construcciones con el si anafórico}

\subsection{Las formas reflexivas}

En este trabajo supondremos que todos los clíticos se generan en una posición determinada, una posición de adjunción al nudo Flex; cuando el verbo se mueve a este lugar para incorporarse a los rasgos [ \pm Tiempo] y $[ \pm$ Concordancia] que caracterizan el núcleo funcional, el clítico se apoya fonéticamente en la forma verbal que resulta de la incorporación.

En la posición de adjunción a Flex se genera también el reflexivo si. La peculiaridad sintáctica de este clítico es la de condicionar el tipo de complemento del núcleo Flex al cual está adjunto, de manera que el sintagma verbal (SV) que este exige como complemento no puede presentar ningún argumento en el lugar del especificador; en otras palabras, el si reflexivo suspende la asignación del papel temático al sujeto profundo, cuando este existe en la red del predicado, y cancela sintácticamente el argumento que debería recibirlo. ${ }^{1}$

Para que pueda darse el significado «reflexivo» en el sentido tradicional del término, es decir, el de una acción realizada por el sujeto y que recae en el sujeto mismo, como en I partecipanti si registrano («Los participantes se registran»), el nudo complejo $\left[s i_{\text {refl }}+\right.$ Flex $]$ toma como complemento un SV necesaria-

1. No es este el único caso de cancelación del sujeto profundo. Para la absorción del papel temático destinado al sujeto que lleva a cabo el participio pasivo, Cfr. Noam CHOMSKY, Lectures on Government and Binding, Dordrecht: Foris Publications, 1981; y Luigi BuRZIO, Italian Syntax. A Government-Binding Approach, Dordrecht: Reidel, 1986, entre otros.

Cfr. también Adriana BELLETTI, «Los inacusativos como asignadores de caso», en Violeta Demonte y Marina Fernández Lagunilla (eds.), Sintaxis de las lenguas románicas, Madrid: Ediciones El Arquero, 1987, p. 197. La autora hace notar que en la oración del francés Il y a un homme à l'appareil ("Hay un hombre al teléfono») y en la del alemán Es gibt einen Mann ("Hay un hombre») aparecen los verbos avoir ("haber, tener») y geben ("dar») que, como todos los verbos transitivos, poseen un papel temático que asignar a la posición del sujeto profundo; sin embargo, en este tipo de construcciones existenciales la presencia del expletivo (fr. $i l$ y al. es) muestra que estos verbos no asignan el papel temático al sujeto profundo, sin dar lugar a ninguna agramaticalidad. Concluye la lingüista: «Conjeturemos, entonces, que los verbos avoir y geben pueden verse afectados por algún proceso cuyo efecto es el mismo que el producido sobre un verbo transitivo por la morfología pasiva». 
mente transitivo del cual cancela el sujeto profundo. Esta restricción explica la agramaticalidad del clítico reflexivo tanto con los verbos intransitivos inergativos, que carecen de un objeto directo ( ${ }^{*}$ Mario si sbadiglia. lit. Mario se bosteza), así como con los verbos inacusativos, ${ }^{2}$ que no asignan un papel temático al sujeto profundo ( ${ }^{*}$ Il bambino si nasce. lit. el niño se nace), con los de ascenso ( ${ }^{*}$ Si sembra che cantino bene. lit. se parece que canten bien) y los meteorológicos (*Si piove. lit. se llueve).

El si reflexivo es una anáfora, es decir, no tiene una interpretación intrínsicamente definida sino que depende de un antecedente para su contenido referencial. Como anáfora está ligada por una categoría rectora y comparte con ella los mismos rasgos nominales de género y número. Consideremos estos ejemplos:

(1) I bambini $i_{\mathrm{i}} i_{\mathrm{i}}$ mascherano. «Los niños se disfrazan.»

(2) I bambini raccontarono che pro $_{\mathrm{i}}$ si mascherarono per gioco. «Los niños contaron que se disfrazaron en broma.»

(3) I bambini $i_{\mathrm{i}}$ vorrebbero $\mathrm{PRO}_{\mathrm{i}}$ mascherarsi. «Los niños querrían disfrazarse.»

(4) È facile $\mathrm{PRO}_{\text {arb i }}$ mascherarsi. «Es fácil disfrazarse.»

El antecedente del si reflexivo puede ser un sintagma nominal (SN) léxico o una categoría vacía, a saber, un pro regido por Flex o un PRO no regido. La única restricción se refiere a la persona: como el reflexivo tiene el rasgo [+Persona] y, en el caso de $s i$, se trata de la tercera, esto lo hace incompatible con SSNN que poseen un rasgo distinto $\left({ }^{*} N o i_{\mathrm{i}}\right.$ si $i_{\mathrm{i}}$ nascondemmo dietro gli alberi. lit. nosotros se ocultamos atrás de los árboles).

Otra característica de las formas verbales reflexivas es que el antecedente del $s i$ anafórico necesita ser un sujeto superficial y no puede tratarse de un argumento con otra función sintáctica (*Scrissi una lettera a Paolo e $_{\mathrm{i}} i_{\mathrm{i}}$ raccontai tutto. lit. escribí una carta a Pablo y se conté todo). El hecho de que el antecedente del $s i$ reflexivo sólo puede ser un constituyente con la función de sujeto superficial se debe a que este clítico, que se origina en posición de adjunción al núcleo Flex, en virtud de la relación núcleo-especificador resulta coindizado con el especificador del SFlex, de modo que en él se copian los rasgos nominales del argumento que ocupa este lugar y que llega a constituir, por esta razón, su antecedente.

¿Qué argumento del predicado ocupa la posición del especificador del SFlex para funcionar como el sujeto superficial de la oración y como el antecedente del si reflexivo?

2. Cfr. David Perlmutter, «Impersonal Passives and the Unaccusative Hypothesis», en Proceedings of the Fourth Annual Meeting of the Berkeley Linguistics Society, Berkeley: University of California, 1978, passim. 
Nuestra hipótesis es que, debido a que el nudo complejo $\left[s i_{\text {refl }}+\right.$ Flex $]$ exige como complemento un SV transitivo del cual cancela sintácticamente el sujeto profundo, el único argumento que puede funcionar como el sujeto superficial de una construcción reflexiva es el que se genera en la posición del objeto directo profundo. En otras palabras, en la construcción reflexiva del italiano se presenta obligatoriamente el movimiento del objeto profundo del verbo a la posición del sujeto gramatical, de manera que este argumento se convierte en el antecedente del clítico reflexivo coindizado con el especificador del SFlex, permitiendo su interpretación referencial.

Una prueba sintáctica de que el SN con la función de sujeto superficial de una construcción reflexiva se origina en la posición del objeto directo es el hecho de que puede ser modificado por un participio absoluto y por un participio adjetival:

(5) a. I partecipanti si registrano. "Los participantes se registran.»

b. Registratisi alcuni partecipanti, ebbe inizio la riunione. registrados-se algunos participantes, tuvo inicio la reunión «Después de haberse registrado algunos participantes, tuvo inicio la reunión.»

c. Tutti i partecipanti registratisi sono stranieri. todos los participantes registrados-se son extranjeros «Todos los participantes que se han registrado son extranjeros.»

En efecto, el participio adjetival sólo puede predicarse de un participante que corresponde al argumento directo del verbo del cual deriva morfológicamente ${ }^{3}$ por lo tanto, en el ejemplo (5b) el SN alcuni partecipanti («algunos participantes") no puede corresponder al sujeto profundo del verbo registrare («registrar»), sino a su objeto directo.

En todas las construcciones en las que interviene un si reflexivo y un SN existe un único argumento, constituido por la cadena de coindización ${ }^{4}$ de la cual forman parte tanto el SN como la anáfora si ligada a ella: I partecipanti $i_{\mathrm{i}} s i_{\mathrm{i}}$ registrano ("Los participantes se registran»). Los dos elementos de la cadena se generan en lugares distintos de la Estructura-P, sin embargo, constituyen un único argumento formalmente discontinuo que se refiere a un único participante, y esto es lo que produce el efecto semántico de redundancia que caracteriza este tipo de construcciones.

¿Qué consecuencia tiene el presente análisis del $s i$ reflexivo a la luz de la Teoría del Filtro del Caso y de la Teoría de los Papeles Temáticos?

El Caso es una necesidad de la cadena de coindización formada por el clítico reflexivo y su antecedente, debido a que esta contiene un argumento del

3. Beth LEVIN y Malka RAPPAPORT, «The Formation of Adjectival Passives», Linguistic Inquiry, n. 17, 1986, passim.

4. Luigi RIzZI, "On Chain Formation", en Borer, HaGIT (ed.), Syntax and Semantics, vol. 19, The Syntax of Pronominal Clitics, Orlando: Academic Press, 1986, passim. 
verbo. El requisito del Caso es satisfecho por el núcleo Flex con el rasgo [+Tiempo], que asigna el nominativo al SN en la posición del sujeto superficial ( $L u i$ (NOM) si lava. "Él se lava»); por consiguiente, también el si reflexivo lo recibe en cuanto parte de la cadena de coindización.

Algo análogo puede decirse con respecto al papel temático. Este es asignado a la posición del complemento del verbo, es decir, la del objeto profundo, ocupada por la huella dejada por el SN en su camino hacia el especificador del SFlex; es la huella, por lo tanto, la que recibe el papel temático de PACIENTE asignado directamente por el verbo. El SN y el clítico, que ocupa una posición no argumental, reciben el mismo papel temático por el hecho de pertenecer, junto con la huella, a la misma cadena de coindización ([I partecipanti $i_{\mathrm{i}}\left[\right.$ si $_{\mathrm{i}}$ registrano $\left.\left.\left.\mathrm{h}_{\mathrm{i}}\right]\right]\right)$. Al formarse la cadena de coindización [I partecipanti $i_{\mathrm{i}} s \mathrm{~h}_{\mathrm{i}} \mathrm{h}_{\mathrm{i}}$, el clítico reflexivo liga la huella del $\mathrm{SN}$ en la posición originaria del objeto profundo y, a su vez, es ligado por su antecedente en la posición del sujeto superficial. ${ }^{5}$

En conclusión, la cadena formada por el si reflexivo, su antecedente y la huella de este recibe el Caso nominativo asignado al SN que llena el especificador del SFlex, y recibe también el papel temático de PACIENTE asignado a la posición del objeto directo profundo donde el SN se genera.

A continuación se propone la siguiente Estructura-P para la construcción reflexiva La bambina si guarda («La niña se mira»):

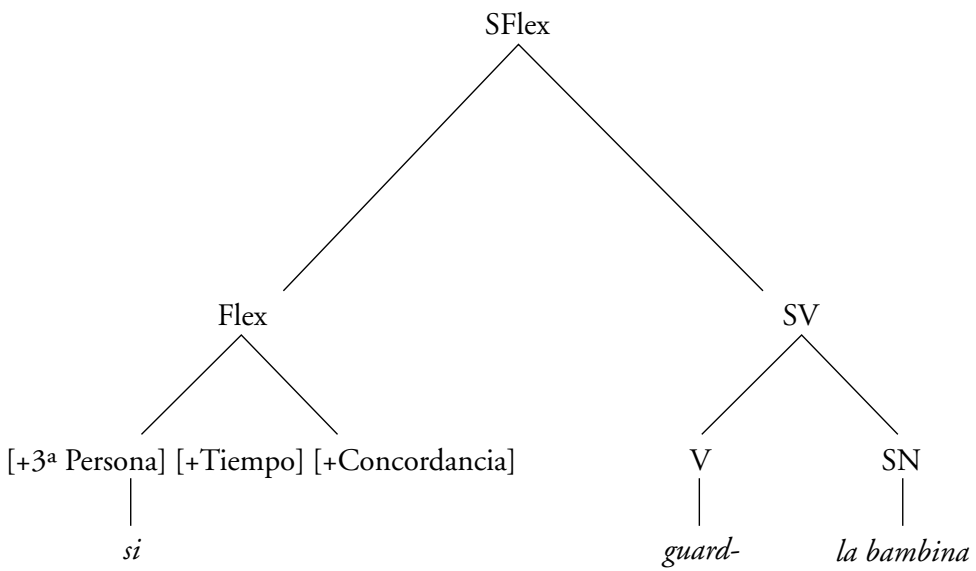

y la siguiente Estructura-S:

5. Las formas $m i$ («me»), $t i$ («te»), $c i$ («nos») y $v i$ («os») pueden funcionar como pronombres reflexivos y también como pronombres acusativos o dativos. En el primer caso ligan la huella del SN que se ha movido desde la posición del objeto profundo al especificador del SFlex $\mathrm{y}$, a su vez, son ligados por este, que constituye su antecedente $\left(\left[T u_{\mathrm{i}} t i_{\mathrm{i}}\right.\right.$ guardi $\left.\mathrm{h}_{\mathrm{i}}\right]$ «Tú te miras»); al contrario, cuando funcionan como pronombres acusativos o dativos ligan un pro en la posición argumental del objeto, pero no son ligados por ningún antecedente ([Maria tii guarda proi] «María te mira». [Maria ti $i_{\mathrm{i}}$ parla pro $_{\mathrm{i}}$ ] «María te habla»). 


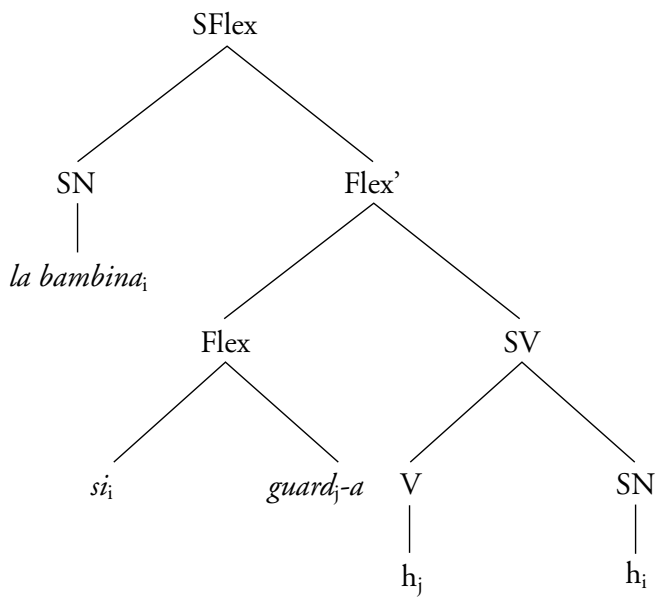

Las construcciones consideradas hasta ahora se caracterizan por un significado reflexivo en el sentido tradicional del término. Existen, además, otras expresiones que comparten con las reflexivas la característica sintáctica de la coindización entre el clítico si y el sujeto superficial, y que representan lingüísticamente un evento con un agente implicado correferencial con el participante referido. Sin embargo, a diferencia de aquellas, este otro tipo de construcciones no se forma siempre con predicados transitivos y puede tener más de un participante.

Por ejemplo, una expresión con un significado recíproco ${ }^{6}$ Due bambini si sorridono («Dos niños se sonríen») se forma con un verbo intransitivo inergativo y en ella interviene, intuitivamente, un objeto indirecto. Si aplicamos las pruebas sintácticas que en el italiano permiten identificar el objeto directo profundo - a saber: la pronominalización con el ne partitivo, la modificación por un participio adjetival, y la construcción con un participio absoluto- descubrimos que el SN que funciona como el sujeto superficial no responde a ellas:

(6) a. Si sorridono due bambini. «Se sonríen dos niños.»

b. ${ }^{*}$ Se ne sorridono due. se de ellos sonríen dos

c. ${ }^{*}$ I bambini sorrisisi sono buoni amici. los niños sonreídos-se son buenos amigos

d. *Sorrisisi i bambini, anche i genitori si salutarono. sonreídos-se los niños, también los padres se saludaron

6. El $s i$ reflexivo y el $s i$ recíproco tienen la misma distribución, con la salvedad de que el recíproco, a diferencia del reflexivo, necesita de un contexto plural. Si este se presenta, como en I bambini si guardano («Los niños se miran») o en I bambini si parlano («Los niños se hablan»), se admite tanto la lectura reflexiva como la recíproca. 
La agramaticalidad del resultado de estas pruebas indica que el sintagma nominal con la función de sujeto superficial no ocupa en la Estructura-P la posición del argumento directo del verbo.

Debido a que el clítico si se genera, como todos los clíticos, en la misma posición de adjunción al nudo Flex, la diferencia entre construcciones como estas y las reflexivas tiene que depender de la posición donde se origina el sujeto superficial. Supondremos que el SN due bambini («dos niños») de la oración (6a) ocupa en la Estructura-P la posición de un argumento interno al SV, de un objeto hermano de V', y que en esta posición recibe del predicado el papel temático correspondiente. ${ }^{7} \mathrm{El} \mathrm{SN}$ que se origina en esta posición estructural se mueve para funcionar como sujeto superficial dejando una huella en el lugar originario. Al situarse en el especificador del SFlex, constituye el antecedente del si anafórico y permite su interpretación referencial.

La Estructura-S que proponemos para este tipo de construcciones es la siguiente:

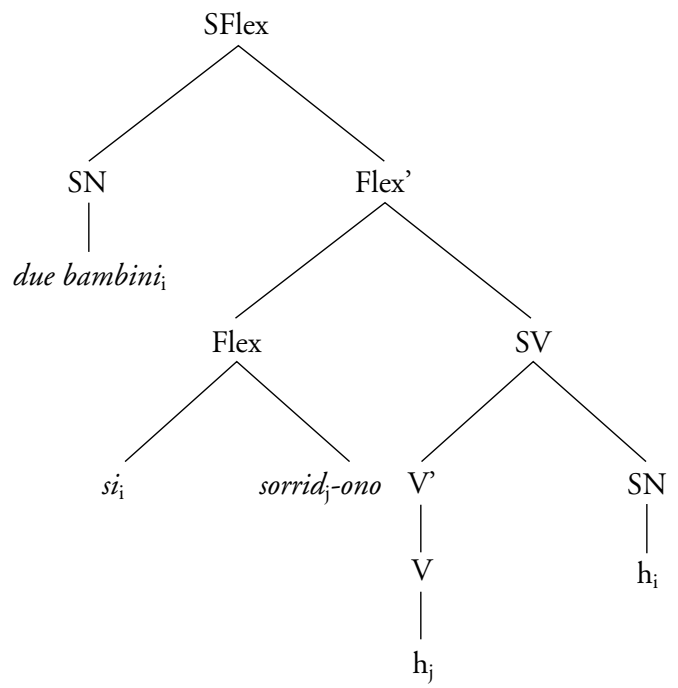

En otras construcciones con el si anafórico puede presentarse también un objeto directo, por ejemplo en Molti bambini si lavano le mani («Muchos niños se lavan las manos»). Al aplicar al SN con la función de sujeto gramatical las

7. En una construcción sin el si reflexivo este argumento se realiza con un sintagma preposicional: La maestra sorride ai bambini («La maestra sonríe a los niños») y, por esta razón, es denominado indirecto. Debido a que este objeto no siempre se realiza, suponemos que a un verbo puede asociarse más de un esquema de asignación de papeles temáticos sin que esto signifique un cambio de significado léxico. 
pruebas sintácticas usadas para identificar el objeto directo profundo de un predicado, obtenemos los siguientes resultados agramaticales:

(7) a. * Se ne lavano le mani molti. se de ellos lavan las manos muchos

b. *I bambini lavatisi le mani erano pochi. los niños lavados-se las manos eran pocos

c. ${ }^{*}$ Lavatisi i bambini le mani, si sedettero a tavola. lavados-se los niños las manos, se sentaron a [la] mesa

La agramaticalidad de estos ejemplos indica que el argumento con la función de sujeto superficial no se origina en la posición del objeto directo, ocupada por el otro argumento de la construcción, que responde, por ejemplo, a la pronominalización con un clítico acusativo (Molti bambini se le lavarono "Muchos niños se las lavaron»). Supondremos, entonces, que se trata de un constituyente hermano de V', dominado inmediatamente por el nudo SV, mientras que el argumento con la función del objeto directo superficial ocupa en la Estructura-P la posición canónica del objeto directo. En otras palabras, estamos frente a una construcción de doble objeto con la posición del sujeto profundo vacía; el verbo marca temáticamente al objeto directo, asignándole el papel temático de PACIENTE, y el constituyente formado por el verbo y su complemento asigna composicionalmente el papel temático de META al segundo objeto. Probablemente, el rasgo [+Persona] es lo que le da al objeto más alto en la estructura la precedencia sobre el objeto directo para funcionar como el sujeto superficial de la oración.

La Estructura-S que proponemos para la oración I bambini si lavano le mani («Los niños se lavan las manos») es la siguiente:

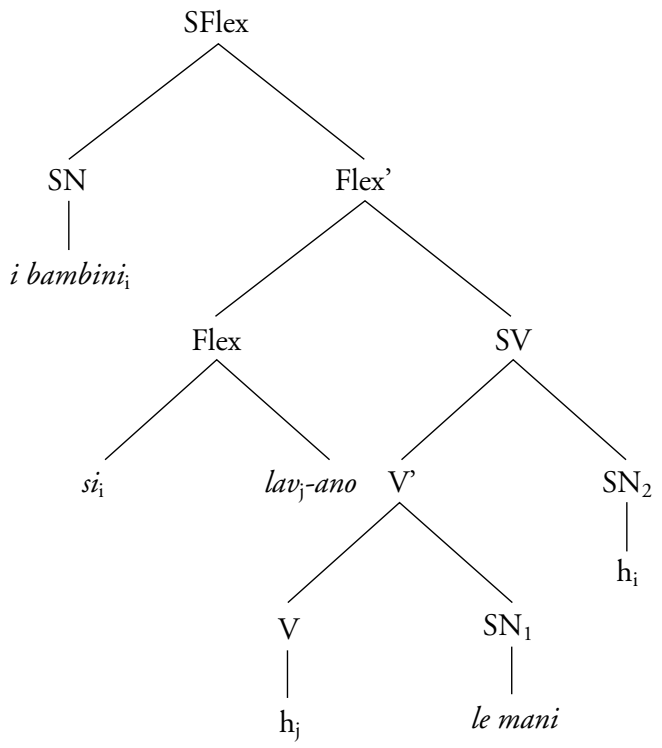




\subsection{Las formas medias}

Pasemos ahora a considerar las construcciones conocidas con la denominación de «medias», "incoativas» o con un "si ergativo»" ${ }^{8}$, que se refieren a eventos espontáneos en cuya realización no interviene ningún agente o causa externos, como Si ruppero pochi bicchieri («Se quebraron pocos vasos»).

Ejemplos como estos excluyen la interpretación reflexiva, en el sentido tradicional del término, no por razones estructurales sino semánticas. El predicado es transitivo y el clítico si lleva a cabo la cancelación sintáctica del sujeto profundo del mismo modo en que lo hace en las expresiones reflexivas; sin embargo, siendo inanimado el único participante referido del evento, este, que es correferencial con el participante cancelado, no puede entenderse como el que realiza la acción que recae sobre sí mismo (es, en todo caso, su causa), y esto es lo que diferencia estas construcciones de las reflexivas.

En todas las construcciones pronominales que se refieren a eventos espontáneos que tienen su sede en el único participante no agentivo, el sujeto superficial tiene el comportamiento sintáctico típico de los objetos directos profundos:

(8) a. Se ne ruppero pochi.

se de ellos quebraron pocos

"Se quebraron pocos de ellos.»

b. I pochi bicchieri rottisi non erano di cristallo.

los pocos vasos quebrados-se no eran de cristal "Los pocos vasos que se quebraron no eran de cristal.»

c. Rottisi i bicchieri, non sapevo come servire lo sciampagna. quebrados-se los vasos, no sabía cómo servir el champán «Una vez que se quebraron los vasos, no sabía cómo servir el champán.»

La Estructura-S de la oración I bicchieri si rompono («Los vasos se quiebran») es la siguiente:

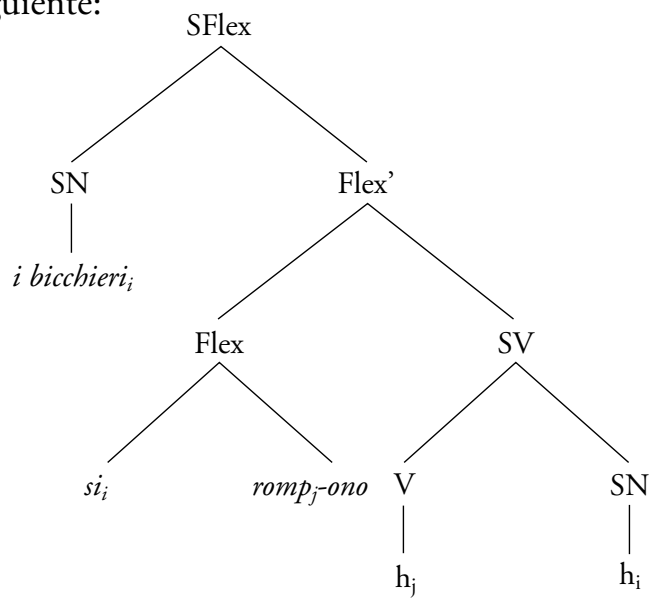

8. Luigi BuRZIO, Italian Syntax. A Government-Binding Approach, Dordrecht: Reidel, 1986, p. 36-42. 
Se han considerado hasta ahora varias construcciones con el si anafórico. Resumiendo, el requisito para su formación es que el verbo tenga, por lo menos, un objeto interno al SV para que pueda asumir la función de sujeto superficial y servir de antecedente para la interpretación referencial de la anáfora.

Cuando el si no establece una relación de coindización con el sujeto gramatical se presenta un tipo distinto de construcciones sintácticas que serán examinadas en el próximo apartado.

\section{Las construcciones con el $s i$ no anafórico}

El clítico si puede presentarse también en construcciones que se caracterizan por un sentido impersonal. Estas construcciones del italiano pueden agruparse, con base en la naturaleza de su sujeto gramatical, en dos subclases: las impersonales sin un sujeto referencial y las impersonales con un sujeto referencial.

\subsection{Las construcciones impersonales sin un sujeto referencial}

Las expresiones impersonales sin un sujeto referencial pueden formarse con un verbo intransitivo inergativo (Si tossisce per il fumo. «Se tose por el humo») o con un transitivo (Si affitta appartamenti. «Se renta departamentos»).

Supondremos que el $s i$ impersonal, a la par que el $s i$ reflexivo o medio, se genera en una posición de adjunción a Flex, donde clitiza en el verbo que se incorpora a los rasgos propios de este núcleo funcional. El si impersonal condiciona de igual modo que el otro si el comportamiento sintáctico de Flex, así que el nudo complejo $\left[s i_{\text {imp }}\right.$ + Flex] toma como complemento un SV sin un sujeto profundo; si el verbo tiene un papel temático que asignar a esta posición, la asignación es suspendida y la realización sintáctica del argumento correspondiente cancelada. Esto resulta muy claro en las construcciones con verbos intransitivos inergativos y transitivos, donde existe incompatibilidad entre el si impersonal y el argumento con el papel temático de AGENTE ( ${ }^{*}$ La gente si $i_{\text {imp }}$ tossisce per il fumo. lit. la gente se tose por el humo; *La gente si $i_{\text {imp }}$ affitta appartamenti. lit. la gente se renta departamentos). La agramaticalidad de estos ejemplos se explica por el hecho de que el sujeto profundo no recibe ningún papel temático, lo que origina una violación del Criterio Temático.

Ahora bien, debido a que una oración necesita de un sujeto, un pro expletivo llena la posición del especificador del SFlex. En Si tossisce per il fumo («Se tose por el humo») no puede ser de otro modo ya que el verbo tossire («toser») es intransitivo inergativo y, una vez cancelado sintácticamente el sujeto profundo, carece de otro argumento capaz de aparecer en aquella posición. Por lo que se refiere a Si affitta appartamenti («Se renta departamentos»), donde interviene el verbo transitivo affittare («rentar»), no se verifica el movimiento del objeto directo profundo appartamenti («departamentos»); 
en efecto, este argumento funciona como objeto también en la Estructura-S, según lo revela la pronominalización con un clítico acusativo ( $L i$ si affitta. lit. los se renta «Se los renta»).

Así que en este tipo de construcciones impersonales un pro expletivo tiene que llenar el especificador del SFlex. El pro expletivo no puede constituir el antecedente del clítico $s i$ porque carece de un contenido referencial, razón por la cual no se establece entre ellos ninguna relación de coindización. En otras palabras, el $s i$ impersonal, a diferencia del $s i$ reflexivo o medio, no constituye una anáfora ya que no posee un antecedente que dicta su interpretación referencial.

De todos modos, las construcciones con el $s i$ no anafórico, a pesar de no tener un sujeto temático, hacen referencia a una persona arbitraria. Como este contenido no puede ser proporcionado por el expletivo nulo, tiene que ser el efecto semántico de la presencia del clítico si. En efecto, este no está desprovisto por completo de contenido. Al igual que el si anafórico, se caracteriza por un rasgo nominal intrínseco, el de [ $+3^{\text {a }}$ Persona], aunque, al no tener ni el rasgo de número ni el de género, el contenido de la tercera persona resulta indeterminado. Esto sugiere que el clítico no anafórico, en última instancia, afecta la concordancia verbal. Veamos de qué modo.

En italiano la concordancia de un verbo finito expresa sincréticamente la persona y el número, y el paradigma de la flexión verbal no posee una terminación específica para una categoría no referencial como el expletivo. Esto significa que, cuando este elemento asume la función de sujeto superficial de una oración, como sucede en las construcciones con el $s i$ impersonal que estamos examinando, el verbo finito, que concuerda siempre con la categoría que llena el especificador del SFlex, manifiesta una concordancia por default en la tercera persona singular con el pro expletivo.

Ahora bien, la presencia del $s i$ no anafórico permite interpretar esta concordancia verbal por default en un determinado sentido; actúa, en otras palabras, como un «modificador» de aquella proporcionando el contenido relativo a una tercera persona indeterminada en número y género. De esta manera el $s i$ no anafórico llega a ser la expresión sintáctica de la persona no especificada, que no está disponible en el paradigma de la flexión personal; se trata, en suma, de una forma sintáctica supletiva de la concordancia personal. ${ }^{9}$

Esto predice que el $s i$ impersonal, debido al rasgo [+Persona], puede aparecer en las oraciones finitas, es decir, en aquellas donde existe un núcleo Flex caracterizado por [+Concordancia], incluyendo el rasgo [+Persona], con el cual el clítico es compatible, y que es agramatical en las oraciones de infinitivo, gerundio o participio, en las que el núcleo funcional carece de este rasgo. Los datos comprueban esta predicción: Qui non si fuma («Aquí no se fuma»); *Qui non può fumarsi (lit. aquí no puede fumarse); ${ }^{*}$ Fumandosi si rischia di ammalarsi (lit. fumándose se corre el riesgo de enfermarse).

9. Guglielmo Cinque, "On si Constructions and the Theory of Arb», Linguistic Inquiry, n. 19, 1988, passim. 
Por lo que se refiere a la construcción impersonal transitiva Si affitta appartamenti («Se renta departamentos»), donde la no promoción del objeto profundo hace que en la posición del sujeto superficial aparezca igualmente un pro expletivo, el si no anafórico tiene la misma función de proporcionar el contenido de la persona indeterminada y de permitir la interpretación impersonal de la concordancia verbal por default.

En conclusión, las construcciones impersonales con los verbos intransitivos inergativos y transitivos que acabamos de considerar tienen en común la presencia de un pro expletivo en la posición del sujeto superficial, es decir, poseen un sujeto no referencial. Proponemos la siguiente Estructura-S para una construcción impersonal formada con un verbo intransitivo inergativo como $\mathrm{Si}$ tossisce («Se tose»):

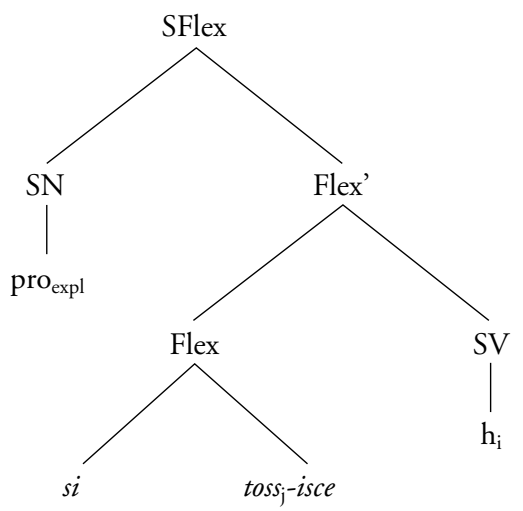

\subsection{Las construcciones impersonales con un sujeto referencial}

Pasemos ahora a analizar el segundo tipo de construcciones impersonales, aquellas caracterizadas por la presencia de una categoría con contenido referencial, que asume la función de sujeto gramatical de la oración. Consideremos, por ejemplo, que el si impersonal puede coocurrir con un verbo inacusativo: Si parte domani («Se sale mañana»). Al no asignar los verbos inacusativos un papel temático al sujeto profundo, el si impersonal no lleva a cabo ninguna cancelación sintáctica de este argumento. Por otra parte, el si no puede suprimir complementos del verbo porque provocaría una violación del Principio de Proyección; supondremos, por lo tanto, que da lugar a la aparición de una categoría vacía en la posición estructural del único argumento del verbo, es decir, el objeto directo profundo. Se trata de un pro arbitrario que, a falta de un sujeto profundo, asume la función de sujeto superficial satisfaciendo, de este modo, el requisito de la predicación. Al igual que en las construcciones impersonales con un pro expletivo, tampoco en este caso existe una coindización entre el si y la categoría vacía que 
llena el especificador del SFlex, razón por la cual esta no constituye el antecedente de aquel.

Por lo que respecta al pro arbitrario con función de sujeto gramatical, ninguna terminación verbal específica para la persona indeterminada está disponible en el paradigma de la flexión; esto significa que la concordancia del verbo se realiza, una vez más, por default, en la forma de la tercera persona singular, así que la función del si no anafórico es, una vez más, la de proporcionar el contenido referencial de la persona indeterminada, dictando la lectura impersonal de toda la construcción.

Una situación análoga a la del predicado inacusativo se presenta cuando el verbo es pasivo, por ejemplo Si è invitati alla festa (lit. se es invitados a la fiesta «Se es invitado a la fiesta»). También en este caso un pro arbitrario se genera en la posición del objeto directo profundo y se mueve al especificador del SFlex para funcionar como el sujeto superficial de la oración; una vez más, la interpretación impersonal es hecha posible por la presencia del clítico si no anafórico.

Como ejemplo del tipo de construcción discutida proponemos la Estructura-S de la oración Si parte («Se parte»):

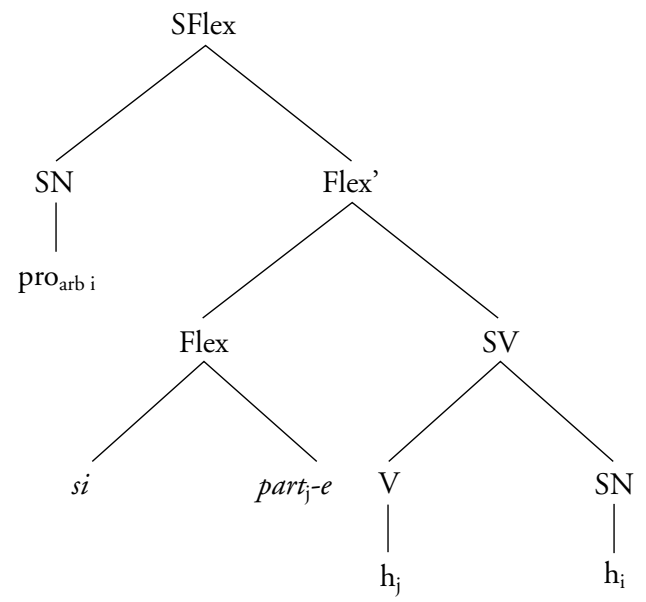

Un último tipo de construcciones impersonales con un sujeto referencial está representado por una oración como Si affittano molti appartamenti ( Se rentan muchos departamentos»). Según lo indica la concordancia verbal, molti appartamenti («muchos departamentos») funciona como el sujeto superficial de la oración; sin embargo, el núcleo del SN se presta a la pronominalización con el ne partitivo (Se ne affittano molti. lit. se de ellos rentan muchos «Se rentan muchos de ellos»), por la razón de que se origina en la posición del objeto directo profundo.

Proponemos la siguiente Estructura-S para la construcción impersonal Si affittano appartamenti ( Se rentan departamentos»): 


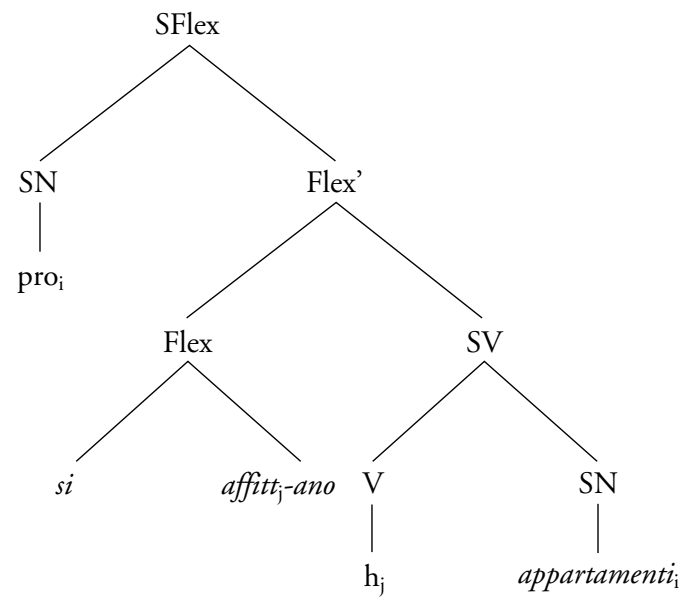

El sujeto postverbal appartamenti («departamentos») está coindizado con un pro en el especificador del SFlex, donde son copiados sus rasgos nominales; por esta razón, el SN recibe in situ el Caso nominativo y el papel temático asignado por el verbo; por otra parte, el pro coindizado permite realizar la concordancia verbal. Lo que dicta el valor impersonal de este tipo de construcciones es, una vez más, la falta de coindización entre el clítico si y el SN que asume la función de sujeto superficial, así que este no puede constituir el antecedente de aquel.

En conclusión, el si impersonal aparece en dos tipos de construcciones sintácticas pero nunca establece un vínculo de coindización con la posición del sujeto superficial, ya sea que esta se llene con un expletivo nulo (cuando el verbo es inergativo intransitivo o transitivo), un pro arbitrario (si se trata de un predicado inacusativo o pasivo) o un SN léxico (si el verbo es transitivo). El clítico sólo posee el rasgo de la tercera persona y, no pudiendo obtener más rasgos nominales de un antecedente, en todas las construcciones en las que interviene aporta exclusivamente el contenido de persona no especificada.

$\mathrm{Al}$ no tener un antecedente, el si no anafórico no está condicionado, como el si anafórico, por la presencia en la red argumental y temática del predicado de un objeto que funcione como el sujeto superficial de la oración (apartados 1.1. y 1.2.), y se caracteriza por una productividad mayor. Su única restricción es la existencia de, por lo menos, un argumento nominal capaz de referirse a un participante humano del evento, razón por la cual es agramatical con los verbos meteorológicos ( ${ }^{*} \mathrm{Si}$ piove. lit. se llueve), que carecen de una red argumental y temática, y con los verbos que subcategorizan un complemento oracional $\left({ }^{*}\right.$ Si sembra lavorare poco qui. lit. se parece trabajar poco aquí; ${ }^{*}$ Si successe che tutti votarono contro. lit. se sucedió que todos votaron en contra). Esta restricción para la aparición del si impersonal es dictada por el rasgo [ $+3^{\text {a }}$ Persona] que, como se vio, aporta el contenido de persona no especificada; por él 
todas las construcciones con el si no anafórico, a pesar de no hacer referencia explícita a un determinado participante - agentivo o no-implican que este existe y que se trata de un humano ( ${ }^{*}$ Si depongono uova. lit. se ponen huevos; * Si affonda lentamente. lit. se hunde lentamente [dicho de barcos]).

A pesar de poseer el rasgo de la tercera persona, el si no anafórico, como se vio, no tiene mayor capacidad referencial. Al generarse en una posición no argumental no constituye, por sí solo, un argumento, ni es parte de él en virtud de una cadena de coindización, de manera que no recibe ni el Caso ni un papel temático. Esto parece contraintuitivo por la razón de que, por ejemplo, las construcciones impersonales con un predicado intransitivo inergativo o transitivo tienen que ver con un participante que realiza la acción; sin embargo, suponemos que en estas expresiones, como también en las oraciones pasivas, el agente está sintácticamente ausente, aunque semánticamente implicado. Se trata, por así decirlo, del efecto semántico de la suspensión del papel temático de AGENTE que, por la presencia del $s i$, no puede ser asignado a ningún argumento, pero sigue perteneciendo a la red temática del predicado.

\section{Conclusiones}

En este trabajo se consideraron dos clases básicas de construcciones con formas verbales pronominales: aquellas con un $s i$ anafórico, que poseen un significado reflexivo o medio, y aquellas con un si no anafórico, que tienen un valor impersonal.

Como todos los clíticos, el si se genera en adjunción al núcleo Flex; si es anafórico se coindiza con la categoría referencial que ocupa el especificador del SFlex y adquiere de ella los rasgos nominales que le hacen falta para identificar a un participante. Al contrario, si el clítico si carece de un antecedente, no obtiene un contenido referencial pleno, de modo que su interpretación queda limitada al valor del rasgo que es suyo intrínsecamente, el de la tercera persona indeterminada; en otras palabras, actúa como un modificador de la concordancia verbal que, por su presencia, se interpreta en sentido impersonal.

La posibilidad de una interpretación reflexiva o media, con un si controlado, y una lectura impersonal, con un si libre, es una prueba de la relativa independencia de este clítico respecto al núcleo Flex y de su condición de adjunto; en efecto, a pesar de que el núcleo Flex se coindiza necesariamente con su especificador, el si puede no hacerlo, de manera que tiene que ser considerado como un elemento aparte de los rasgos de flexión.

Los dos si, el anafórico y el no anafórico, constituyen una única entidad lingüística ${ }^{10}$ que sólo posee el rasgo [+Persona], la tercera; se generan en el

10. Para un análisis unitario del si reflexivo y del si impersonal Cfr. Maria Rita MANZINI, "On Italian Si», en Hagit BORER (ed.), Syntax and Semantics, vol. 19, The Syntax of Pronominal Clitics, Orlando: Academic Press, 1986, p. 241-261; y Luigi BurZIO, «On the Morphology of Reflexives and Impersonals», en Christiane LAEUfER y Terrel A. MORGAN (eds.), Theoretical Analyses in Romance Linguistics, Amsterdam: J. Benjamins, 1992, p. 399-414. 
mismo lugar estructural y condicionan del mismo modo el comportamiento sintáctico del núcleo Flex al que están adjuntos, que toma como complemento un SV sin un sujeto profundo; su única diferencia radica en la relación de coindización que el clítico si puede o no establecer con la categoría que funciona como sujeto superficial.

\section{Bibliografía}

Belletti, Adriana (1981). "Frasi ridotte assolute», Rivista di Grammatica Generativa, n. 6, p. 3-33.

- (1987). «Los inacusativos como asignadores de caso». En: DemOnTe, Violeta; FeRNÁNDEZ LaGUnILla, Marina (eds.), Sintaxis de las lenguas románicas, Madrid: Ediciones El Arquero, p. 167-230.

BOSQUE, Ignacio (1999). «El sintagma adjetival. Modificadores y complementos del adjetivo. Adjetivo y participio». En: BosQue, Ignacio; DemonTe, Violeta (eds.), Gramática descriptiva de la lengua española, vol. 1, Madrid: Espasa Calpe, p. 217-310.

BuRZIO, Luigi (1986). Italian Syntax. A Government-Binding Approach, Dordrecht: Reidel.

- (1992). «On the Morphology of Reflexives and Impersonals ». En: LAEUfER, Christiane; Morgan, Terrel A. (eds.), Theoretical Analyses in Romance Linguistics, Amsterdam: J. Benjamins, p. 399-414.

CHOMsкY, Noam (1981). Lectures on Government and Binding, Dordrecht: Foris Publications.

CINQUE, Guglielmo (1988). "On si Constructions and the Theory of Arb», Linguistic Inquiry, n. 19, p. 521-581.

DEMONTE, Violeta (1990). «Transitividad, intransitividad y papeles temáticos». En: Demonte, Violeta; Garza Cuarón, Beatriz (eds.), Estudios de lingüistica de España y México, Ciudad de México: Universidad Nacional Autónoma de México/El Colegio de México, p. 115-150.

Koopman, Hilda; Sportiche, Dominique (1991). "The Position of Subjects», Lingua, n. 85, p. 211-258.

LEVIN, Beth; RAPPAPORT, Malka (1986). "The Formation of Adjectival Passives», Linguistic Inquiry, n. 17, p. 623-661.

MANZINI, Maria Rita (1986). «On Italian Si». En: Borer, Hagit (ed.), Syntax and Semantics, vol. 19, The Syntax of Pronominal Clitics, Orlando: Academic Press, p. 241-261.

OTERO, Carlos P. (1986). «Arbitrary Subjects in Finite Clauses». En: BORDELOIS, Ivonne; Contreras, Heles; Zagona, Karen (eds.), Generative Studies in Spanish Syntax, Dordrecht/Riverton: Foris Publications, p. 81-109.

Perlmutter, David (1978). «Impersonal Passives and the Unaccusative Hypothesis». En: Proceedings of the Fourth Annual Meeting of the Berkeley Linguistics Society, Berkeley: University of California, p. 157-189.

POLLOCK, Jean-Yves (1989). «Verb Movement, Universal Grammar, and the Structure of IP», Linguistic Inquiry, n. 20, p. 365-424.

RENZI, Lorenzo; SALVI, Giampaolo (eds.) (1991). Grande grammatica italiana di consultazione, vol. 2, I sintagmi verbale, aggettivale, avverbiale. La subordinazione, Bologna: Il Mulino.

RizzI, Luigi (1986). «On Chain Formation». En: Borer, HAGIT (ed.), Syntax and Semantics, vol. 19, The Syntax of Pronominal Clitics, Orlando: Academic Press, p. 65-95. 
SALVI, Giampaolo (2002). "Il problema di 'si' e l'uso riflessivo di essere», Verbum, n. 4, p. 377-398.

STOWELL, Timothy (1983). «Subjects across Categories», The Linguistic Review, n. 2, p. 285-312.

STOWELl, Timothy (1989). «Subjects, Specifiers, and X-Bar Theory». En: Baltin, Mark R.; Krock, Anthony S. (eds.), Alternative Conceptions of Phrase Structure, Chicago: The University of Chicago Press.

Williams, Edwin (1980). "Predication», Linguistic Inquiry, n. 11, p. 203-238.

WiLliams, Edwin (1981). «Argument Structure and Morphology», The Linguistic Review, n. 1, p. 81-114. 